\title{
Towards an Unified Virtual Mobile Wireless Architecture
}

\author{
Oleg Asenov ${ }^{1}$ and Vladimir Poulkov ${ }^{2}$ \\ ${ }^{1}$ The Faculty of Mathematics and Informatics, St. Kiril and St. Metodius University \\ of Veliko Turnovo, 5003 Veliko Turnovo \\ ${ }^{2}$ The Faculty of Telecommunications, Technical University of Sofia, Bulgaria, \\ vkp@tu-sofia.bg
}

Received September 2013; Accepted November 2013

Publication January 2014

\begin{abstract}
In this paper the idea for a new unified wireless network architecture is presented. The need for such a new architecture is justified and its major characteristics are outlined. Natural development of Long Term Evolution (LTE) beyond 4G, network convergence and/or evolution could naturally lead the way towards such a new unified wireless architecture, but it is expected that many of the problems of the current co-existing architectures will co-exist with such scenarios. The proposed idea of the development of a new Unified Virtual Cell Architecture (UVCA) could have the potential to overcome the problems of the mobile wireless networks of today and through the development of a good migration roadmap could be a faster and successful approach.
\end{abstract}

Keywords: Network Convergence, Network Architectures, Long Term Evolution, Wireless Access.

\section{Introduction}

The main questions raised in this paper are related to the evolution of wireless networks and the need of a new unified wireless network architectures and infrastructures. A general overview at the current situation could lead to some simple conclusions. There are many wireless standards such as UMTS, LTE, WiMax, WiFi, Bluetooth, RFID, ZigBee, Packed Radio solutions, etc. In many

Journal of Communication, Navigation, Sensing and Services, Vol. 1, 93-104.

doi: 10.13052/jconasense2246-2120.115

(c) 2014 River Publishers. All rights reserved. 
cases these standards are related to one and the same or overlapping services and applications. Practically this could be considered on the one hand as parallel service development or parallel investment in one and the same application services, in one and the same functionalities and in the different standards. On the other hand the existence of different wireless standards leads to the necessity one device to have installed hardware for several different wireless interfaces.

Obvious thing is also the "saturation", i.e. the availability of parallel infrastructures and dense deployments of access points in one and the same locations delivering one and the same services. In some locations infrastructure density is higher than device density. Besides cost effectiveness this raises many other problems such as throughput, interference and electro-magnetic compatibility problems. Even with the application of new highly effective modulation, channel coding and interference suppression techniques in many places the existing architectures have already reached the throughput limit (Shannon limit).

Another conclusion that could be drawn is related to the strong dependence of users on service providers. Practically a service is available only if the user pays "tax" for the infrastructure, which means that if an access to two services is required, which are offered via two different independent infrastructures, the user has to pay two "taxes infrastructure".

These simple conclusions raise the questions if there is a need for a change in the current telecom architectures and infrastructures and if there are driving forces that are strong enough to push forward to such a change. For most of the developments in telecommunications the major drivers are related to the manufacturers of new devices, the developers of new applications and services, customer demands, business competition, costs, complexity and regulations.

Manufacturers of new devices and the developers of new applications constantly release newer and smarter user devices, new applications and application-driven platforms and services. These novel communicating devices and machines introduced in the network generate more and more demands and new requirements on the infrastructure. The internet enables the everyday generation of new applications and services, without any fundamental constraints to delivering them anywhere in the world to any user using any device, thus requiring from the telecommunication networks higher and higher data transmission speeds.

During the last years mobile applications and mobile social networking have surpassed pure voice services. There are problems with the ability to 
process the volume of data transmitted by end users, especially in larger urban areas. Broadband mobile users are expected to reach 3.4 billion by 2014 , $60 \mathrm{~b}$ in 2020 . The result is an overall increase of the multimedia traffic in the wireless networks. As a consequence, Traffic Engineering and Quality of Service (QoS) issues are becoming more and more complex. Multimedia services require a new way of traffic engineering, differentiated treatment of data flows based on traffic type, QoS, customer Service Level Agreements (SLA), user activity and user preferences. Implementation of scenarios for dynamic resource management, advanced carrier aggregation, power control, etc., with competing system operators is constrained because of complexity. This all is putting enormous pressure for changes in the design of the wireless networks architecture and infrastructure.

The overall pricing of services and cost model of the networks infrastructure is also under consideration. Today users are not concerned about technological details. Their main concern is to obtain cheaper and cheaper services that are not constrained by time and location (i.e. anytime and anywhere). To satisfy customer demands network operators strive to differentiate themselves from competitors by offering unified or better functioning products. In addition to this network operators tend to become pure Service Providers (SP). The dominant SPs move towards a dataonly network, carrying VoIP. They are offering voice-only services for "free" as part of bundled data packages. The requirements towards secure, reliable and efficient data transmission, data management and QoS are growing as this is a prerequisite for their domination on the market. This of course requires also good and efficient Operation, Administration, Maintenance and Provisioning (OAM\&P), which brings up the necessity of a more reliable and easier for maintenance infrastructure. Mobile operators are outsourcing OAM\&P and focus on becoming only service providers.

Pressure for changes in the overall model of the infrastructure and architecture of the different types of networks are also lower costs, higher throughput, lower power consumption and green issues such as electromagnetic compatibility and health. Example is the tendency backhaul networks to serve both for fixed and mobile access based on fiber optic technology and Ethernet. With mobile wireless delivering higher and higher throughput, the size of the cells decreases (femto-, pico), thus changing and spreading the fiber network infrastructure to small cell locations. Regulation and standardization also could act as driving forces for changes in the infrastructure, such as the 
proposed European shared-facilities model of "RAN (radio access network) sharing".

Having in mind all the above mentioned, the main question that is raised in this paper is if the change of the network architectures of today will be a natural process of evolution or is it justified to think towards the development of a new unified wireless architecture. The rest of the paper is organized as follows: in the next section two basic evolutionary approaches towards a new architecture are discussed, section III presents the idea of the development

of a unified virtual mobile architecture and some conclusions are outlined in section IV.

\section{Evolution towards a New Wireless Network Architecture}

To meet all future challenges, the expectations from a new mobile wireless architecture are generally related to the development of a big range of the access points - from $5 \mathrm{~m}$ up to $50-100 \mathrm{~km}$. ensuring extreme data rates and high traffic capacity. This supposes full integration of the fixed and mobile infrastructures and full utilization of the benefits of the different types of wireless networks which will bring to overall coverage, security, improved services and reduced costs. From the user perspective such a full integration of infrastructures means that the user will be able to connect to the network anywhere and anytime not depending on the underlying access network and will effectively utilize the infrastructure due to the implementation of a shared, flexible and reliable solution. The user will benefit also from the integration of billing and services. Billing (integrated or through bundles) must become only service dependent and must not have a network component associated with it. The introduction and integration of new services must not depend on types of networks or network architectures. Once a service is deployed over the network, it will become a service that can be run anywhere and anytime over any type of network.

From technological and service provider perspective expectation from a new architecture will give the possibility of modular and flexible territorial spread of the access, based on self-organization, self-configuration and self-regulation. This should be a unified type of dynamic spectrum access ensuring efficient and scalable utilization of the frequency spectrum. A new architecture will be cognitive and intelligent adapting to user requirements, new personalized multimedia services and applications, required QoS, etc. and at the same time "green" and cost effective, i.e. with minimized energy consumption and very-low-cost deployment and maintenance. 
One possible way leading to such a new wireless architecture is the natural evolution of LTE beyond $4 G$. It is expected the unique air interface of LTE, that offers high downlink and uplink speeds, to meet the challenges of the services and the QoS requirements in the future. LTE will continue to evolve and will bring to an overall shift towards such a new network architecture. Cell sites will become smaller thus increasing capacity and lowering power consumption. LTE will become the global and unique technology for future mobile broadband as its underlying architecture is based on IP - fully packet-switched, flexible, with support of different cell sizes ranging from tens of meters, spanning femtocells and picocells to macrocells with up to a 100-km cell radius. LTE will handle the expected massive increase of users and multimedia traffic. Some arguments are given that LTE evolution will create an new LTE ecosystem - "one that is rich and open, and fosters the development of innovative mobile services and applications through the availability of new application programming interfaces which allow developers to have access to specific network assets as well as enablers such as location, presence, and security" [1].

At the first glance simple reasoning is that there should be no reason to deviate from the LTE track. Questions that could arise are related to what extend all the new applications, services and scenarios will be supported sufficiently well by the LTE evolution. Major consideration here is the issue of complexity. Very small cells in the LTE architecture means that the architecture and equipment must support complex wireless Ethernet topologies under the requirements for reliable delivery of real-time services with the necessary QoS, imposing new approaches for overall management, control, reliability and efficiency. There will be other complexity issues such as precise network timing and synchronization. For small-cell IP/Ethernet based networks that do not transport the clock reference transparently providing the necessary QoS, while maintaining delay and jitter under permissible limits to ensure recovery of the clock reference, will be quite a technological challenge [2].

Another way that could lead to a new architecture is the fixed-mobile convergence and the introduction of hybrid architectures for wireless access where the general target is the deployment of a global all-IP wireless/mobile network. Fixed-mobile convergence is practically related to the unification of wireless and wireline voice, video, and broadband data services through a seamless integration of wireless and fixed networks. Such scenarios are already being implemented as they bring advantages not only for end users, but also for service providers and operators. From a user point of view, such 
a convergence will bring unified billing, ubiquitous and seamless connectivity, and access to a consolidated set of services. From an operator point of view there are technical and economic benefits. They are coming from the possibility of a more effective utilization of the resources of the last mile access network and efficient use of the available public and personal, wireless local and metropolitan access infrastructures. One way towards fixed-mobile convergence is the use of fixed broadband access to backhaul mobile traffic from traditional (macro) cell sites or Long Term Evolution (LTE) evolved Node Base (eNB) stations. Another development nowadays is the introduction of smaller cell sites such as the femto cells to extend the coverage and capacity of a wireless network into homes, buildings, and closed areas, or to use hybrid architectures such as LTE and Wi-Fi. The major goal is to increase overall wireless network capacity by off-loading traffic from the fullsize base stations and enabling much higher bit rates than when connected to a macro base station. The economic benefits are related to the cost-effective use of the existing, fixed broadband access network in combination with the high speeds and low latency afforded by LTE, which will enable service providers to offer integrated wireline and wireless broadband services, while lowering their operations costs. The growing demand for low-cost mobile broadband is driving the process of fixed-mobile convergence and the development of heterogeneous networks in which different radio access technologies will co-exist $[3,4,5]$.

The main question that is raised when considering convergence is how much time will it take and will it bring to a radical change in the networks architecture as there are many technical and other problems to be solved. Key challenges are the air interface, the architecture and the protocol convergence of the different technologies. It is difficult to design a converged air-interface for different types of wireless networks due to the different coverage and channel conditions. Besides, the different networks have different signal processing capability, and different bandwidth allocation schemes. In a converged architecture the complexity should be evaluated to achieve an acceptable trade-off between the cost and performance gain. For a really converged network architecture, air-interface, protocol and control signaling should be tightly converged. In such a converged network, MAC and network layer protocols should be jointly optimized. In converged networks, the downlink and uplink control signaling should be designed, and some "cross-MAC" designs need to be implemented at the gateway. A different resource allocation scheme should be considered for converged networks, especially 
for scenarios where there is large number of wireless nodes with heavy traffic [6].

A related issue concerns convergence ideology. This is based on the principle of achieving the converged infrastructure through maximum reuse of the available technologies which practically hampers, or in the best case delays the creation and implementation of yet another technology. Other problems are related to the convergence of the infrastructure including costly OAM issues which have to be solved. As all the different types of future networks should be service-oriented, new usage scenarios have to be developed which could hardly be implemented directly into a converged or heterogeneous network architecture and infrastructure. Actually many of the problems of the current co-existing architectures will also coexist in a convergence scenario and will not be solved for a long period of time.

\section{Migration towards a New Unified Wireless Architecture}

Considering the problems with network convergence a possible approach is the migration towards a new wireless network architecture. The gains from a new architecture are expected to be similar to those what we gained from cable internet: access anytime and anywhere; much more consolidated and cost-effective investments in the quality of the access infrastructure; decrease of prices of access and services; increase of the overall effectiveness in the industrial segment, due to the lower expenses for OAM\&P of the infrastructures. This new architecture could be focused on Services, Applications and Content, will incorporate the architectures of device-to-device and machine-to-machine types of communications, and will drive the path towards Future Internet.

The general characteristics that such a new unified architecture must have are:

To have a new unique and unified infrastructure for wireless access, organized through clear rules for hierarchy, territory, frequency allocation.

To fully implement the proposed European shared-facilities model of "RAN (radio access network) sharing" with shared infrastructure, shared backhaul, and shared cells.

To be accessed via one unified interface in the terminal equipments computers, tablets, smart phones, and why not also industrial controllers, identification tags, etc.

To be fully IP based, including mobile telephony. 
To be self-planning, self-organizing, self-monitoring, self-regulating architecture in respect of power control, automatic switch-off of zones or groups of access points when no users or requests for service are available allowing for significantly reduced emphasis on manual intervention.

To be flexible, cognitive, intelligent in order to ensure dynamical development and evolution and employ all the advantages of cognitive radio networks.

To be fully cross-layer designed architecture with the possibility of "virtualizing" different networks and substructures (operators, private networks, etc.) in order to avoid the proliferation of various types of small-cell infrastructure equipment.

There are many challenging tasks that have to be solved for the practical implementation of such a process of migration towards a new unified architecture. Some of the challenges related to Research and Development are:

R\&D of a cognitive, self-reconfigurable, self-optimizing, architecture depending on location, user activity and required QoS.

R\&D of an Unified Virtual Cells Architecture (UVCA), build over the existing multi operators' Physical Cells (Base Stations) architectures. An interesting task will be the definition of optional secondary role-based virtualization of the UVCA by: QoS, by Privacy (Private UVCA), by availability for requesting temporary services (On-Demand UVCA). In this aspect another issue will be to ensure the transparency and continuity of services provided on secondary role-based virtualization trough a set of Unified Virtual Cells. The way to such a Unified Virtual Cells Architecture is to aggregate the coverage and services provided by the multi operators' Physical Cells based on one territory. This should not be a "mechanical" aggregation; a Physical Cells "Team Working Algorithm" (TWA) must be taken under R\&D. During the first step of migration to a UVCA, the Physical Cells TWA will work in parallel with previous conventional features under user selection, such as the choice to be a unified user or operator dependent user. The second step will be setting-up the secondary role-based virtualization for unified use, but the option for operator depended use will be still available. At the third step there will be only unified use.

R\&D of a Unified Virtual Base Station as a basic granule of UVCA based on the aggregation of the existing physical set of base stations and on the Physical Cells TWA - which will be the core algorithm of UVCA. 
R\&D of a technology for self-organization, self-monitoring and self-control, for an energy saving "green" infrastructure.

R\&D of methods for virtualization of the existing technologies and services to be used for different applications (virtual applications) and also for group access to different services (virtual operators).

R\&D of Media Access method enabling migration to multi-access domains and permitting optional or conditional user subscriptions to different access sub-domains.

Besides the R\&D challenges there will be many other issues to be resolved such as the formulation of new technical conditions and requirements for spectrum use and allocation. New models of ownership and management of resources including, but not limited to, combinations and bundles of spectrum, equipment, processing, storage and energy resources, must be proposed. The architecture must be developed in such a way in order to ensure a clear plan for migration from the existing wireless architectures towards a Unified Virtual Mobile Wireless Architecture (UVMWA).

Possible roadmap for the practical implementation of the UVMWA is the following:

Stage 1. Establishment of a management Layer of the UVMWA, including primary \& secondary UVMWA border session controllers (servers) per unified SOA area;

Stage 2. Attachment of the mobile operators" "Soft Switches" (switch environment) to the management layer;

Stage 3. Upgrade of base station and wireless access points middle ware with the SOA agent for UVMWA;

Stage 4. Upgrade the user wireless terminals OS with SOA client for UVMWA;

Stage 5. Start Up the UVMWA hybrid services;

Example of Start Up procedure: $\{. \ldots \ldots$. Primary Border Session Controllers On Service; ? Primary Border Session Controllers Interconnect; ? Operators VOIP Soft Switches On Service; ? Base Station \& Wireless Access Points Service Path On Service; ? SOAAgents for UVMWA to Primary Border Session Controllers On service; ? User Equipment Configuration; ? Offering Hybrid Services for End Users.

Stage 5. Parallel Operation: Traditional and UVMVA;

Stage 6. Switch to UVMWA. 


\section{Conclusion}

In this paper the idea for the migration towards a unified wireless architecture is discussed. The main questions raised are related to the necessity and the driving forces for such a new architecture. The major arguments in its favor are related to the facts that the available mobile services and mobile contents are growing faster than mobile networks. The result will be that the building up of different parallel wireless data infrastructures for users to access same services and content could never be under consideration. Users, manufactures and service providers will be major drivers towards such a new architecture. Users want to be connected anytime and anywhere, while manufacturers of mobile devices, operators and service providers want to make the cost savings. Network convergence and/or evolution could lead the way towards such a new unified wireless architecture, but the (proposed in this paper) idea of the development of an Unified Virtual Cell Architecture has the potential for a faster migration. The development of a good migration roadmap with several

stages could be a successful approach to new generation mobile networks beyond 4G, with novel approaches to resource management, traffic engineering and meeting the future challenges of multimedia services and QoS requirements.

\section{References}

[1] Harstead E., Menendez H. The-Evolution-and-Promise-of-LTE. OSP Magazine, 2012. http://www.ospmag.com/issue/article/The Evolution and Promise-of-LTE

[2] Mudoi U. Understanding Small-Cell Unification's Vital Role In LTE And 4G. August 02, 2012.

[3] Fixed and Mobile Networks: Substitution, Complementarity and Convergence, OECD Digital Economy Papers, No. 206, OECD Publishing (2012). http://dx.doi. org/10.1787/5k91d4jwzg7b-en.

[4] Cellular and Wi-Fi: A Match Made in Heaven? Signals Ahead. March 2012, Vol. 8 No. 4.

[5] Raj M., Narayan A., Datta S., and Sajal K. Das. Fixed Mobile Convergence: Challenges and Solutions. IEEE Communications Magazine December 2010; pp. 26-34.

[6] Zhang J., Shan L., Hu H, and Yang Yang. Mobile Cellular Networks and Wireless Sensor Networks: Toward Convergence. IEEE Communications Magazine March 2012; pp.164-169. 


\section{Biographies}

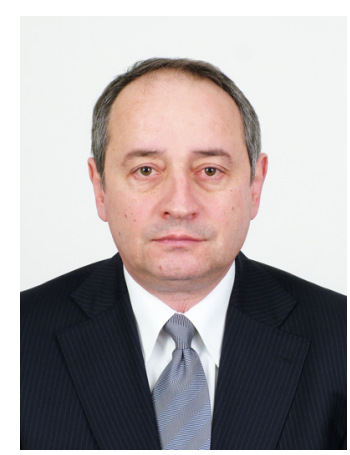

Professor Vladimir Poulkov PhD, has received his MSc and PhD degrees at the Technical University of Sofia. He has more than 30 years of teaching and research experience in the field of telecommunications. The major fields of scientific interest are in the fields of Information Transmission Theory, Modulation and Coding. His has expertize related to interference suppression, power control and resource management for next generation telecommunications networks. Currently he is Dean of the Faculty of Telecommunications at the Technical University of Sofia. Senior Member of IEEE.

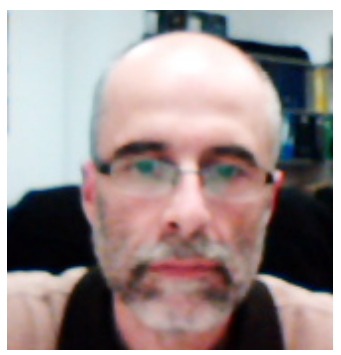

Associated Professor Oleg Asenov, has received his MSc degree at the Technical University of Gabrovo and $\mathrm{PhD}$ degree at the Technical University of Sofia. He has more than 20 years of teaching and research experience in the field of Telecommunications. The major fields of scientific interest are modeling, simulation and design of computer networks based on graph theory and applied heuristics algorithms. Currently he is Associated Professor at the St.Cyril and St.Methodius University of Veliko Tyrnovo, Member of IEEE. 
